\title{
BOSQUE DE ROBLE O PLANTACIÓN DE CONIFERAS, ¿QUÉ PREFIEREN LOS LÍQUENES EPÍFITOS?
}

\section{Oak forest or conifer plantation, what do epiphytic lichens prefer?}

\author{
Diego Simijaca', Bibiana Moncada² y Robert Lücking ${ }^{3}$
}

Simijaca, D., Moncada, B. y Lücking, R. (2018). Bosque de roble o plantación de coníferas, ¿qué prefieren los líquenes epífitos? Colombia Forestal, 21(2), 123-141

Recepción: 11 de noviembre de 2017

Aprobación: 22 de marzo de 2018

\section{Resumen}

Quercus humboldtii es un elemento dominante en los bosques altoandinos de Boyacá, Colombia, el cual, a pesar de ser una especie sombrilla, registra consumos per-cápita de $1300 \mathrm{Kg}$.año ${ }^{-1}$ y el remplazo de sus rodales por plantaciones de Pinus patula. Este remplazo afecta las comunidades liquénicas más sensibles. Para rastrear dichas afecciones se examinaron los líquenes en cuatro árboles de cada especie forófita. Cada árbol fue dividido en cinco estratos verticales y se midió la rugosidad, humedad, acidez y luminosidad en cada estrato. Los análisis comprendieron índices de diversidad, tablas Anova y escalas multidimensionales. Se registraron 161 especies de líquenes, se reconocen 53 especies exclusivas sobre pino y 45 exclusivas de roble. Se destacan Cladonia ceratophylla y Herpothallon rubrocinctum como especies comunes entre forófitas, y la exclusividad de Astrothelium spp, Anzia leucobatoides y Leptogium diaphanum sobre los robles. La luminosidad y la rugosidad son factores de influencia en la distribución de los líquenes.

Palabras clave: bosque nativo, corteza, cianolíquenes, composición, forófito.

\begin{abstract}
Quercus humboldtii is a dominant element in the high Andean forests of Boyacá, Colombia. Despite being an umbrella species, it has a per-capita consumption of $1300 \mathrm{Kg} . y e a r^{-1}$ and its natural stands are widely replaced with plantations of Pinus patula. This replacement affects the most sensitive epiphyte communities. To track these conditions, lichens were examined on four trees of each phorophytic species. Each tree was divided into five vertical levels and bark roughness and acidity, as well as humidity and luminosity were measured at each level for each tree. The data were analysed by means of diversity indexes, Anova, and multidimensional scaling. 161 lichen fungal species were recorded, 53 of these unique to pine trees and 45 unique to oak trees. Cladonia ceratophylla and Herpothallon rubrocinctum stand out as a frequent species shared between both phorophytes, whereas Astrothelium spp, Anzia leucobatoides y Leptogium diaphanum are exclusive to oaks. Luminosity and bark roughness are factors influencing lichen community formation.
\end{abstract} Keywords: native forest, bark, composition, cyanolichens, phorophyte.

\footnotetext{
Universidad Distrital Francisco José de Caldas. Bogotá, Colombia. dfsimijacas@correo.udistrital.edu.co Autor para correspondencia 2 Universidad Distrital Francisco José de Caldas. Bogotá, Colombia. Ibmoncada@udistrital.edu.co

3 Botanical Garden and Botanical Museum Berlin. Berlin, Alemania. rluecking@bgbm.org
} 


\section{INTRODUCCIÓN}

Los bosques de roble en Colombia se distribuyen desde los 750 hasta los $3450 \mathrm{~m}$ de altitud y ocurren en cerca de 18 departamentos del país, con lo que se reconocen como elementos importantes de los bosques andinos de Colombia (Avella y Cárdenas, 2010). Sin embargo, su dominancia es más evidente en localidades que superan los 2500 $\mathrm{m}$ de altitud, en las cuales Quercus humboldtii Bonpl. se convierte en un elemento típico del paisaje (Marín-Corba y Betancur, 1997).

Estos bosques ofrecen una gran variedad de hábitats para mamíferos, aves y organismos invertebrados (Gasca e Higuera, 2008; Otálora, 2003; Ramírez y Pérez, 2007; Sáenz-Jiménez, 2010; Vanegas, Fagua y Flórez, 2012), que a su vez contribuyen con la dispersión de semillas y, de tal manera, con el enriquecimiento de la flora en la cual orquídeas, bromelias, helechos, briófitos y líquenes también son conspicuos sobre los troncos y ramas de $Q$. humboldtii (Alzate, Cardona y Callejas, 2001; Ariza et al., 2010; Ávila, Ángel y López, 2010; Gil y Morales-Puentes, 2014; Vargas-Rojas y Morales-Puentes, 2014) .

Pese a su función como especie sombrilla, los robles han sido históricamente explotados por la calidad de su madera y la versatilidad de usos que puede dársele a la misma (Ariza et al., 2010). Ejemplo de esto son los departamentos de Boyacá y Santander, lugares donde $Q$. humboldtii se utiliza en la fabricación de cabos para herramientas y para el uso doméstico como combustible en la elaboración de alimentos, alcanzando un consumo per-cápita superior a los 1300 Kg.año ${ }^{-1}$ (Avella y Cárdenas, 2010; Medina 2010). Otro impacto de las actividades humanas en los robledales recae en la deforestación para ampliar la frontera agrícola o para el establecimiento de plantaciones forestales (Kapelle, 2006). Con lo cual se registra una pérdida de 28 550ha de bosques de roble hasta el año 2006 (CAS, 2006), que son remplazados por cultivos y pastos; mientras, en el caso de las plantaciones forestales, el área aumenta visiblemente con
53 195ha de Pinus patula Schltdl. \& Cham. para el año 2002 (FAO, 2002).

El cambio en el uso del suelo y el remplazo de los bosques nativos por plantaciones de coníferas trae consigo fuertes repercusiones que afectan la calidad del suelo, la circulación tanto de nutrientes como de agua, además de la dinámica de las poblaciones de plantas y animales que habitan estos ecosistemas (Barlow et al., 2007; Calviño-Cancela, Rubido-Bará y van Etten, 2012; Goward y Arsenault, 2000; Hietz-Seifert, Hietz y Guevara, 1996), debido a que se homogenizan las condiciones ambientales, como luz y humedad, acidez y rugosidad de los árboles a nivel local, con lo que se suprime la variedad de microecosistemas ofrecida por los bosques naturales (Käffer, Ganade y Marcelli, 2009; Ardila, Moncada y Lücking, 2015).

Los líquenes corresponden a uno de los grupos de organismos notablemente afectados por la paridad en las condiciones ambientales y con frecuencia son usados como indicador para el estudio del estado de las comunidades epífitas. La homogenización de la riqueza, la dominancia de especies generalistas y la desaparición de ciertos grupos funcionales son respuestas comunes ante esta transformación del paisaje (Goward y Arsenault, 2000; Bäcklund Jönsson, Strengbom, Frisch y Thor 2016; Berg, Hunt y Gordon, 2013; Calviño-Cancela, López de Silanes, Rubido-Bará y Uribarri, 2013; Ardila et al., 2015). De hecho, se ha demostrado la alta sensibilidad de los líquenes a condiciones microclimáticas como la luz incidente y la humedad (Lücking, 1999; Soto, Lücking y Rojas, 2012) y las características propias de los forófitos, como: la arquitectura del dosel, la estructura y acidez de la corteza, su rugosidad o la presencia de ornamentos y lenticelas (Cáceres, Lücking y Rambold, 2007; Hauck, 2011; Käffer, Ganade y Marcelli, 2009; Rosabal, Burgaz y Reyes, 2013). Esta sensibilidad ha permitido el uso de los líquenes como bioindicadores en diversos aspectos ambientales que incluyen: la calidad del aire (Rubiano y Chaparro, 2006; Llop, Pinho, Matos, Pereira y Branquinho, 2012; Simijaca, Vargas y Morales, 2014), 
el cambio en el uso del suelo (Stofer et al., 2006; Maestre et al., 2011; Giordani, Brunialti, Bacaro y Nascimbene, 2012) y la fragmentación de bosques (Rivas Plata, Lücking y Lumbsch, 2008; Pulido y Ramos, 2016; Ramírez-Morán, León-Gómez y Lücking, 2016).

El estudio de la flora liquénica en las plantaciones forestales de Colombia tiene como referente una investigación que utilizó la familia Graphidaceae como elemento bioindicador y comparó la diversidad liquénica de un bosque de roble con una plantación de Eucalyptus globulus Labill. (Ardila et al., 2015). De otro lado, la flora liquénica de los bosques altoandinos de Colombia ha sido ampliamente documentada y es objeto de constante actualización (Sipman y Aguirre-C, 1982; Aguirre-C, 2008; Moncada, Coca y Lücking, 2013; Lücking et al., 2016). Sin embargo, son escasos los estudios que evalúan la preferencia de forófitos y las condiciones que presenta cada hospedero intrínsecamente para albergar líquenes epifitos (Soto et al., 2012; Ardila et al., 2015).

Un sector de especial interés para el estudio de la biota liquénica corresponde al corredor de robles Guantiva-La Rusia-Iguaque, el cual es considerado el corredor con los bosques de roble más extensos en el territorio colombiano al abarcar 141293 ha y transcurrir por 22 municipios de los departamentos de Boyacá y Santander (Avella y Cárdenas, 2010). Esta característica le confiere especial importancia a $Q$. humboldtii porque es soporte para múltiples especies de líquenes epífitos, dentro de las que se destacan algunas nuevas especies para la ciencia dentro los géneros Cora y Sticta que, a su vez, son indicadores de bosques conservados (Lücking et al., 2016; Moncada et al., 2013).

Un caso de estudio en el corredor Guantiva-La Rusia-Iguaque la reserva de la sociedad civil Rogitama Biodiversidad (Arcabuco- Boyacá), que corresponde a un predio privado en el que, durante más de 30 años, se ha hecho un esfuerzo por la restauración del bosque de roble y en el cual el proceso de recuperación del bosque nativo se ha llevado a cabo alterno a la plantación de rodales de Pinus patula, sobre la cual no se realiza tala. Esta reserva sustenta una gran diversidad de flora y fauna representativa del corredor de robles y es el albergue de especies de plantas y animales en peligro de extinción como Chalybea macrocarpa (Uribe) Morales-P. \& Penneys (Gil-Leguizamón, Morales-Puentes y Díaz-Pérez, 2014) y Coeligena prunellei Bourcier (Chavarro, 2005). Adicionalmente, es el refugio de insectos como Pseudopogonogaster iguaquensis Salazar y Carrejo, que utiliza líquenes como superficie de mimetismo (Parada, 2015). No obstante, en los bosques de la reserva no se han realizado estudios concernientes a los líquenes epífitos y al efecto que la generación de un "bosque mixto" pueda tener en la distribución de las comunidades liquénicas. Por consiguiente, esta investigación tiene por objeto comparar la riqueza y composición de la biota liquénica asociada a $Q$. humboldtii y $P$. patula en la reserva natural Rogitama Biodiversidad, con el fin de reconocer el impacto del establecimiento las comunidades liquénicas y determinar si las características propias de los forófitos como rugosidad, acidez, luminosidad incidente y humedad varían entre las especies de forófitos y tienen efectos en la riqueza y composición de las comunidades de líquenes.

\section{MATERIALES Y MÉTODOS}

\section{Área de estudio}

El estudio se llevó a cabo en la reserva de la sociedad civil Rogitama Biodiversidad, un área protegida privada, localizada en la vereda Peñas Blancas del municipio de Arcabuco (Boyacá), 547'47.3" $\mathrm{N} ; 7^{\circ} 26^{\prime} 56.0^{\prime \prime} \mathrm{W}$. Altitudinalmente se ubica entre los 2485 a $2530 \mathrm{~m}$ de altitud y presenta una temperatura promedio anual de $13{ }^{\circ} \mathrm{C}$, con un régimen de precipitación bimodal de 1900 mm.año ${ }^{-1}$ (Gil-Leguizamón, Morales-Puentes y Díaz-Pérez, 2014). La reserva abarca 29 hectáreas de las cuales 22 son de bosque nativo y dentro de estas, ocho 
hectáreas presentan robledales. Por otro lado, seis hectáreas son plantaciones de P. patula; y una hectárea es dedicada a la vivienda (Chavarro, 2005). La importancia estratégica de esta área protegida está soportada en su ubicación en el corredor conservación Guantiva-La Rusia-Iguaque, una de las áreas más representativas en la protección de $Q$. humboldtii (Morales et al., 2007).

\section{Selección de forófitos y recolección de ejemplares}

Se seleccionaron y marcaron cuatro árboles de $P$. patula y cuatro de $Q$. humboldtii con altura superior a $5 \mathrm{~m}$ y circunferencia a la altura del pecho (CAP) superior a $100 \mathrm{~cm}$, que presentaran ramas resistentes al ascenso (Gradstein, Nadkarni, Krömer, Holz y Nöske, 2003). Para registrar el mayor número de especies por forófito, cada árbol se dividió en cinco estratos de acuerdo con la zonificación planteada por Johansson (1974) y se ascendió mediante técnicas de escalada simple con una sola cuerda (Lowman, Moffett y Rinker, 1993). Cada levantamiento se realizó en un transecto vertical, con un flexómetro de $100 \mathrm{~cm}$ en cada estrato, en los que se recolectaron los líquenes que estaban debajo de la cinta métrica y se anotaron las características morfológicas y de ubicación espacial para cada ejemplar recolectado. El transecto vertical se replicó en cada punto cardinal. Para la extracción del material del dosel externo se usó un corta ramas que permitió el acceso y desmote de las porciones más externas. Las muestras de líquenes recolectadas y etiquetadas se secaron por exposicion al ambiente $y$, posteriormente, se llevaron al herbario forestal Gilberto Emilio Mahecha Vega (Sección Criptógamas) UDBC, donde se realizó el correspondiente proceso de determinacion mediante claves taxonómicas (Coca y Sanín, 2010; Lücking, Aptroot, Chaves, Sipman y Umaña, 2008; Moncada y Forero, 2006; Moncada, 2012) y la aplicación de pruebas químicas con $\mathrm{NaClO}$, $\mathrm{KOH}, \mathrm{KI}$ y parafenilendiamina, además del uso de esterescopio Leica TS1232W26 y microscopio
Olympus CX31 con los cuales se realzó la observacion y el corte de las estructuras reproductivas de los liquenes para la observacion de caracteres utiles en la identificación taxonómica.

\section{Registro de características de los forófitos}

Para cada estrato vertical se midieron los $\mathrm{pH}$ de la corteza mediante la extracción de un fragmento de la misma y la maceración de 0.5 g diluidos en $5 \mathrm{ml}$ de agua destilada, que se dejaron ocho horas en reposo para su posterior análisis en laboratorio con un medidor de pH digital RoHS (Kricke, 2002). Se examinó la estructura de la corteza en cada estrato, tomando como referencia un testigo de 10 centímetros, medida que se modificó de la metodología de Rosabal, Burgaz, Altamirano y Aragón (2012), quienes usan un testigo de $5 \mathrm{~cm}$. La intensidad de luz relativa (luminosidad) se registró para cada forófito con el uso de fotografías periféricas de cada levantamiento con un lente ojo de pez de $180^{\circ}$, que se transformaron a blanco y negro y se calcularon los porcentajes de cada color mediante el programa Gap Light Analizer 2.0 (Frazer, Canham y Lertzman, 2000), con el color blanco como la luz relativa y el negro como la cobertura del dosel. La humedad relativa se registró puntualmente en el levantamiento con el uso de un psicrómetro digital EHM-10.

\section{Análisis de datos}

Se calculó la diversidad alfa $(\alpha)$ para cada especie forófita mediante los índices de riqueza, dominancia de Simpson y equidad de Shannon-Wiener (Magurran, 1988). Se evaluó la relación entre la riqueza y los estratos verticales mediante una correlación de Spearman y la diversidad beta mediante el uso del índice de Jaccard (Magurran, 1988). Para evaluar el comportamiento de las variables ambientales en cada hospedero se realizó un análisis de varianza Anova no paramétrica (Kruskal-Wallis). Para reconocer la distribución de las comunidades y la influencia de las variables en 
la distribución de los líquenes epífitos se hizo el análisis de escalamiento multidimensional no paramétrico (NMS) (McCune, Grace y Urban, 2002).

\section{RESULTADOS}

\section{Riqueza liquénica de las especies forófitas}

Se recolectaron 964 muestras de líquenes en las dos especies hospederas, de las cuales 903 se identificaron hasta nivel de especie; mientras las otras 61 muestras se identificaron a nivel de género por carecer de estructuras que permitieran su determinación. Dicha revisión permitió el reconocimiento de 161 especies agrupadas en 40 géneros y 24 familias (anexo 1). Las familias con mayor riqueza fueron Parmeliaceae (ocho géneros-64 especies), Graphidaceae (8-31) y Physciaceae (2-12).

Los arboles de Pinus patula (pino), con 116 especies, exhibieron una mayor riqueza liquénica que la observada en Quercus humboldtii (roble), con 108 especies (tabla 1). Para las dos especies forófitas se observan bajos valores de dominancia (Simpson; $\mathrm{D}^{\prime}=0.02$ ) y valores similares en la equidad con $\mathrm{H}^{\prime}=4.15$ para P. patula y $\mathrm{H}^{\prime}=4.18$ para $Q$. humboldtii. Se observa una composición liqúenica que difirió sustancialmente entre las dos especies forófitas con un índice de Jaccard $\left(\mathrm{I}_{\mathrm{J}}=0.38\right)$ que señala que se comparten el $38 \%$ de las especies.

Tabla 1. Índices de diversidad para los forofitos evaluados

\begin{tabular}{lcc}
\hline \multicolumn{1}{c}{ Índices } & $\begin{array}{c}\text { Pinus } \\
\text { patula }\end{array}$ & $\begin{array}{c}\text { Quercus } \\
\text { humboldtii }\end{array}$ \\
\hline Riqueza de especies & 116 & 108 \\
Simpson $\left(\mathrm{D}^{\prime}\right)$ & 0.02 & 0.02 \\
Shannon- Wienner $\left(\mathrm{H}^{\prime}\right)$ & 4.15 & 4.18 \\
\hline
\end{tabular}

En el caso de los pinos, Parmotrema mellissii (C.W. Dodge), Herpothallon rubrocinctum (Ehrenb.) Aptroot, Lücking \& G. Thor y Heterodermia japónica (M. Satô) Swinscow y Krog fueron las especies más frecuentes (anexo 1). En contraste, Crocodia aurata (Ach.) Link, Erioderma sorediatum Galloway y Jørg y Parmotrema indicum Hale, entre otras, presentan un solo registro y hacen parte de las 50 especies únicas del pino.

En los robles, Herpothallon rubrocinctum, Hypotrachyna bogotensis (Vain.) Hale y Cladonia ceratophylla (Sw.) Spreng fueron las especies más frecuentes (anexo 1) y se reconocen 47 especies únicas de este forófito, como son Heterodermia corallophora (Taylor) Skorepa, Hypotrachyna densirhizinata (Kurok.) Hale y varias especies de Astrothelium, que también se destacaron por ser especies raras con un solo registro.

\section{Caracterización fisicoquímica y microambiental de los forófitos}

Respecto a la rugosidad, se encontró una mayor heterogeneidad en los registros obtenidos sobre las cortezas de $P$. patula comparado con Q. humboldtii (figura 1a) y se observan diferencias significativas entre los forófitos (Kruskal-Wallis, $p=0.006$ ) que indican que los pinos (promedio $=12.58$ ) eran más rugosos que los robles (promedio $=10.60$ ). En lo concerniente al $\mathrm{pH}$, los robles se consideraron significativamente más ácidos que los pinos (Kruskal-Wallis, $p<0.05$ ). Aunque la luminosidad fue más heterogénea en pinos que en robles (figura 2c), no presentó diferencias significativas (Kruskal-Wallis, $p=0.279$ ). Por su parte, la humedad tampoco registró diferencias significativas entre las especies forófitas (Kruskal-Wallis, $p=0.279$ ).

\section{Distribución espacial de las comunidades liquénicas}

La ordenación de las muestras resultó en dos agrupaciones visiblemente separadas, lo que muestra una correlación significativa con la especie de forófito. Se observa que la distribución de las comunidades liquénicas está relacionada con las diferencias en los parámetros de rugosidad, luminosidad y $\mathrm{pH}$ entre pinos y robles, mientras la humedad es el 

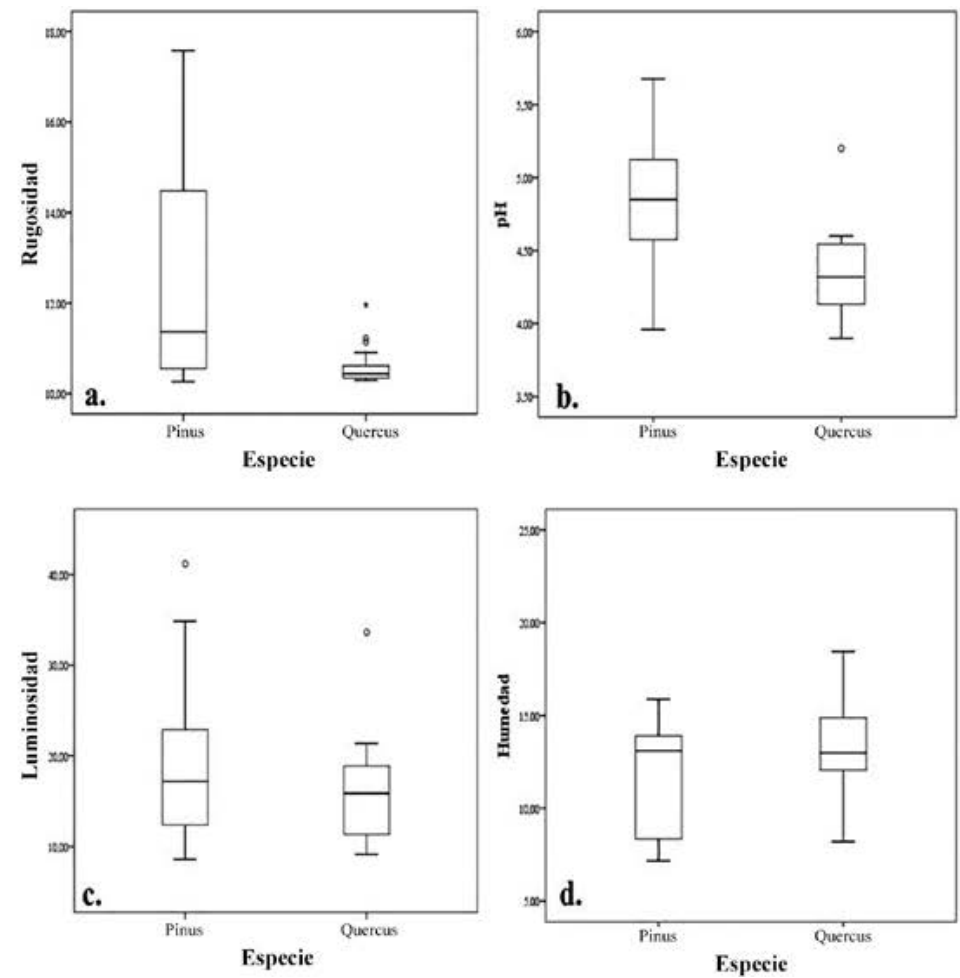

Figura 1. Diagramas de caja para los factores fisicoquímicos (a. rugosidad; b. pH) y microambientales, (c. Luminosidad; d. Humedad) que muestran la variabilidad en las especies forófitas.

parámetro de menos influencia dada su posición perpendicular en el diagrama. Se reconocen tres muestras de Pinus patula dentro de la agrupación de Quercus humboldtii, que corresponden con las especies compartidas por los dos forófitos.

\section{DISCUSIÓN}

\section{Impacto de las plantaciones de pino que remplazan bosque de roble}

Los resultados de esta investigación ponen en evidencia el impacto sobre la composición de la biota liquénica que ocurre al remplazar el bosque nativo de Q. humboldtii por plantaciones de P. patula. Se observó que, aunque los índices de diversidad alfa en ambas especies eran similares, su composición varió notablemente con el cambio de forófito. El impacto también se percibe en la cantidad de especies particulares que presenta cada hospedero (50 especies particulares del pino y 47 especies particulares del roble). Además, en el robledal se encontraron especies de las familias Graphidaceae (thelotremoides) y Lobariaceaeae, las cuales son indicadoras de bosques bien conservados (Cáceres et al., 2007; Rivas Plata et al., 2008; Soto et al., 2012; Ramírez-Morán et al., 2016), mientras que en la plantación de pino dominan las especies de Parmotrema, Hypotrachyna y algunas especies de Graphidaceae (lirelados) como Graphis elegans y Phaeographis inconspicua, que son afines a condiciones más hostiles en los sustratos como contaminación atmosférica, alta incidencia de la luz, presencia de resinas en la corteza de los forófitos (Mossmann, Weidner y Martins, 2012) y que para el caso de Graphidaceae se destacan como especies pioneras en cortezas de fácil desprendimiento como la de Eucalyptus globulus (Ardila, Moncada y Lücking, 2014; anexo 1). 
Estas modificaciones en la diversidad y estructura de las comunidades liquénicas parecen ser un patrón común al comparar bosques nativos con plantaciones forestales, en las que se han encontrado cambios en la riqueza, abundancia y composición de los líquenes como respuestas frecuentes a la intervención (Estrabou, 2007; Calviño-Cancela et al., 2013; Nascimbene, Thor y Nimis, 2013; Bäcklund et al., 2016). Por ejemplo, Ardila et al. (2014) reconocieron un impacto sustancial en la estructura de las comunidades de especies de la familia Graphidaceae, que cambian al contrastar un robledal característico de los bosques altoandinos colombianos con una plantación de Eucalyptus globulus. Los mismos autores encontraron riquezas homogéneas entre los árboles de eucalipto evaluados y la separación de las comunidades liquénicas en los forofitos exóticos y los de bosque de roble; esto último coincidiendo con los resultados de los análisis de escalamiento multidimensional realizados.

De manera similar Käffer, Ganade y Marcelli (2009) detectan cambios en la composición liquénica de los bosques nativos de Araucaria en Rio Grande do Sul, comparándolos con plantaciones de pino y eucalipto. El cambio más notable se observó en términos de la dominancia de especies umbrófilas, que se presentan recurrentemente en los bosques nativos de Araucaria, mientras en las plantaciones dominan los líquenes heliófilos. En los resultados obtenidos se identificaron cianolíquenes de los géneros Coccocarpia, Erioderma, Leptogium y Sticta, considerados como especies umbrófilas y más frecuentes en los robles; mientras que en los pinos son frecuentes las especies heliófilas de los géneros Parmotrema, Hypotrachyna y Usnea.

Los resultados obtenidos representan no solamente un cambio en las especies asociadas a cada forófito, sino también en los grupos funcionales característicos de los líquenes (Koch Mossmann, Martins, Lucheta y Müller, 2013; Lakatos, Rascher y Büdel, 2006; Llop et al., 2012; Stofer et al., 2006), lo que a su vez se relaciona con la variedad de microclimas que pueden encontrarse en los árboles de Rogitama, en los que características como la rugosidad, la luminosidad y la acidez de las cortezas varían entre los forófitos (figura 2). Por consiguiente, se esperaría que los rodales de pino presentaran condiciones más hostiles para el crecimiento de cianolíquenes, por presentar cortezas ácidas, sitios con amplia incidencia de luz y un régimen homogéneo de humedad, tal como lo han registrado varios estudios (Bäcklund et al., 2016;

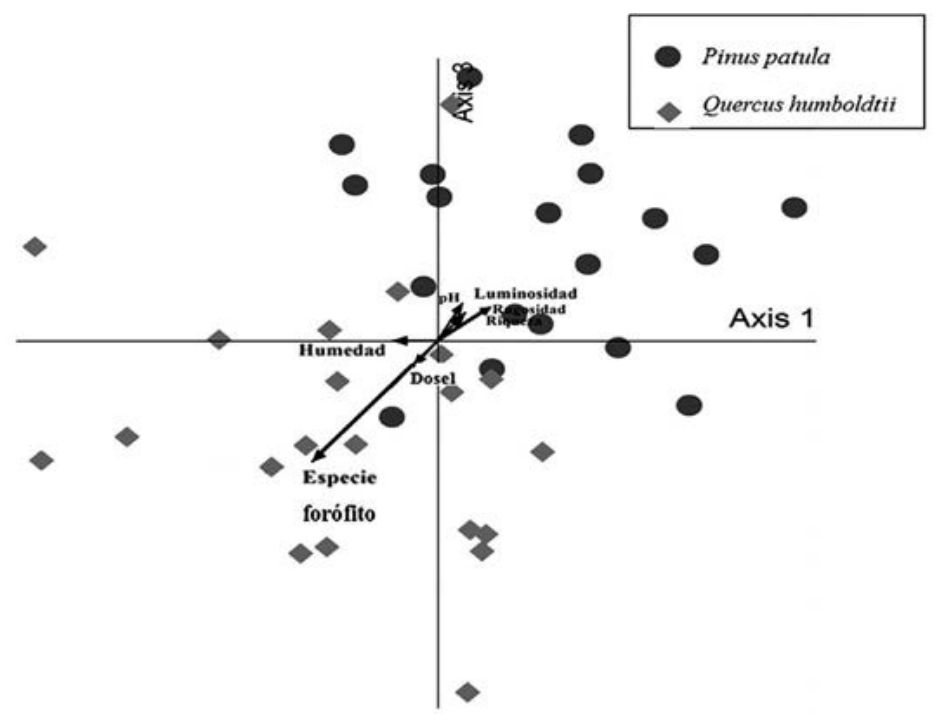

Figura 2. Diagrama de ordenación para la composición de los espacios muestreados en las dos especies de forófitas. 
Calviño-Cancela et al., 2013; Käffer, Ganade y Marcelli, 2009). En contraste, las condiciones evidenciadas y la presencia exclusiva de Erioderma sorediatum sobre el pino implican que en ocasiones este forófito pueda considerarse como facilitador para el desarrollo de especies umbrófilas, lo que corrobora lo indicado por Ellis (2012) y Giordani et al. (2012).

A pesar de los cambios en la composición, que se producen con el establecimiento de las plantaciones, es importante evaluar la dominancia que ejercen las especies resilientes al disturbio. En lo referente a los líquenes, en nuestra investigación se registran varias especies de la familia Parmeliaceae, como: Hypotrachyna costaricensis (Nyl) Hale, Hypotrachyna osorioi (Hale) Hale, Parmotrema arnoldii (Du Rietz) Hale y Parmotrema simulans (Hale) Hale que son frecuentes en pinos y robles. Esto concuerda con algunos estudios los cuales sugieren que varias especies de la familia Parmeliaceae son tolerantes a la desecación, acidificación de sustratos e incluso a la presencia de material particulado (Hauck y Javkhlan, 2009; Käffer, Ganade y Marcelli, 2009; Llop et al., 2012; Simijaca et al., 2014). Esta familia también es dominante sobre varios forófitos en los bosques del Chaco argentino, donde crecen con exuberancia (Estrabou, 2007). Los bosques altoandinos colombianos también son referentes en la diversidad de Parmeliaceae, donde se registran como una de las familias de mayor riqueza, junto con Lobariaceae y Physciaceae (Aguirre-C, 2008; Ramírez-Morán et al., 2016; Simijaca, 2011; Wolf, 1993b).

En el caso de los taxones exclusivos, se destaca la presencia de la familia Trypetheliaceae y el género Astrothelium (cuatro especies), como taxón particular de los robles de este estudio. En contraste con las formas lireladas, características de la familia Graphidaceae, que abundan en los pinos (anexo 1). Al respecto, se han delimitado algunos biotipos que reflejan el estado de conservación o intervención de los bosques (Koch Mossmann et al., 2013; Mercado-Díaz, Gould, González y Lücking, 2015; Ramírez-Morán et al., 2016), donde morfotipos telotremoides y con peritecios, representan los bosques bien conservados; mientras algunas formas lireladas de la familia Graphidaceae concuerdan con el biotipo de bosques intervenidos (Rivas Plata et al., 2008). De manera similar, el género Sticta es más diverso en robles que en pinos y puede relacionarse con los biotipos descritos por Ramírez-Morán et al (2016) para los bosques conservados.

Con estas diferencias, los líquenes anteriormente mencionados pueden ser utilizados como indicadores de continuidad ecológica (Rivas Plata et al., 2008), en la que Anzia, Astrothelium, Leptogium y Sticta son habitantes de los robledales y se sugieren como biotipos de los bosques bien conservados; mientras que Cladonia, Herpothallon, Hypotrachyna y Parmotrema son característicos de la plantación de pino y se sugieren como biotipos de los bosques intervenidos. Lo anterior concuerda con algunos registros de Ramírez-Morán et al. (2016) y Pulido y Ramos (2016), que incluyen tanto características morfológicas como fisiológicas de los líquenes en la delimitación de los biotipos.

No obstante, la presencia de cianoliquenes como Coccocarpia prostrata y Erioderma sorediatum y su exclusividad en el pino, sugieren que buenas prácticas de manejo de las plantaciones o el mantenimiento de remanentes sin tala y entresaca de las mismas, conservan algunas condiciones ambientales que son favorables para estos grupos de líquenes sensibles (Calviño-Cancela et al., 2013) y pueden promover el recambio por especies de Lobariaceae o Trypetheliaceae que son frecuentes en los bosques conservados (Moncada, 2012; Ramírez-Morán et al., 2016).

De otro lado, las modificaciones en los patrones de diversidad como consecuencia de la intervención humana no son manifestaciones exclusivas de los líquenes, pues la flora vascular del sotobosque y del dosel también experimentan cambios considerables con la transformación del bosque nativo (Alzate, Cardona y Callejas, 2001; Braun et al., 2017; Marín-Corba y Betancur, 1997). Del mismo modo, la reducción de hábitat y alimento, 
producto de la homogenización de recursos en las plantaciones, afecta especies de aves (Barlow et al., 2007; Calviño-Cancela et al., 2013; Sáenz-Jiménez, 2010), mamíferos (Otálora, 2003; Ramírez y Pérez, 2007; Sáenz-Jiménez, 2010) y artrópodos (Gasca e Higuera, 2008; Parada, 2015; Vanegas, Fagua y Flórez, 2012). Por ejemplo, Macroagelaius subalaris Boissonneau, Chorostilbon poortmani Bourcier y C. prunellei son aves con distribución restringida a los robledales del corredor Guantiva-La Rusia-Iguaque (del cual hace parte Rogitama) que se ven afectadas por la explotación de madera en departamentos como Boyacá y Santander, trayendo como consecuencia la reducción en sus poblaciones, con la transición de bosques nativos a plantaciones forestales (Avella y Cárdenas, 2010; Sáenz-Jiménez, 2010).

Los resultados de este estudio reiteran el éxito en el uso de los líquenes como indicadores en diversos aspectos ambientales, dentro de los que se destacan la medición de la calidad del aire (Llop et al., 2012; Rubiano y Chaparro, 2006; Simijaca et al., 2014) y el cambio en la salud de los bosques (Dingová Košuthová y Šibík, 2013; Nascimbene et al., 2013; Song, Liu y Nadkarni, 2012). Teniendo en cuenta que modificaciones en parámetros como la acides del sustrato, la rugosidad del mismo y la luz incidente sobre los tallos liquénicos modifican la composición de las comunidades de dichos organismos y permiten rastrear la preferencia de los mismos por determinada condición ambiental.

\section{¿Que prefieren los líquenes?}

Particularmente para Pinus patula se destacan especies exclusivas como Chrysothrix candelaris, Graphis cognata, G. elegans y Heterodermia leucomela, que han sido mencionados por algunas investigaciones dentro de los morfotipos resistentes a condiciones ambientales hostiles y en este estudio se presentan sobre las cortezas más rugosas y con mayor incidencia de la luz. No obstante, otras de sus exclusividades corresponden a Coccocarpia prostrata y Erioderma sorediatum que corresponden a especies que según Goward y Arsenault (2000) son poco frecuentes sobre coníferas y prosperarían en condiciones de baja luminosidad.

Por su parte, como especies únicas de Quercus humboldtii se observa a Anzia leucobatoides (que corresponde a un nuevo registro para el país), Leptogium diaphanum y Sticta isidioimpresula y tres especies de Astrothelium que corresponden a especies propias de bosques conservados y denotan una preferencia por ambientes de baja luminosidad, pero que son tolerantes a la acides que la presencia de taninos le confiere a los robles (Martínez-Gil et al., 2017). Estos resultados son comparables con los estudios Wolf (1993a), que señalan entre 35 y 50 especies en las formaciones vegetales evaluadas y con las 41 especies de Graphidaceae sobre robles, identificadas por Ardila et al. (2015). Además, con bosques de Weinmannia tomentosa del municipio de Guasca-Cundinamarca en el que se registran 54 especies (Ramírez-Morán et al., 2016).

Es importante señalar que la preferencia de los líquenes por determinado forófitos depende de un conjunto de parámetros dentro de los cuales la rugosidad y la acides de las cortezas y la luminosidad son determinantes en la distribución de las comunidades liquénicas, corroborando lo mencionado por Cáceres et al. (2007) y Soto et al. (2012). Por consiguiente, algunas especies como Chrysotrix candelaris prefirieron las cortezas rugosas y la alta luminosidad presente en los rodales de pino, mientras otras especies como Anzia leucobatoides fueron más afines a cortezas lisas y con acides pronunciada por la presencia de taninos como es el caso del roble, que también presenta una mayor cobertura del dosel.

Dadas las similitudes en la metodología utilizada y la riqueza de la reserva Rogitama, se considera superior a la encontrada por Cornelissen y Ter Steege (1989) en las tierras bajas de Guyana. También es comparable con el reconocimiento de 250 especies en un bosque de tierras bajas en Venezuela (Komposch y Hafellner, 2000) y con 
la identificación de 150 especies en el estado de Alagoas al noreste de Brasil (Cáceres et al., 2007). Ubicando a la reserva Rogitama como un resguardo de flora liquénica en el cual el esfuerzo de conservación del robledal y las buenas prácticas de manejo de los rodales de pino permiten que se comparta el $38 \%$ de la flora liquénica y que se presenten especies de cianoliquenes sobre las cortezas de pino.

\section{CONCLUSIONES}

Con este estudio se reconoce la reducción en la frecuencia y diversidad de las especies umbrófilas e indicadoras de continuidad ecológica como Anzia leucobatiodes, Astrothelium spp, Leptogium diaphanum y Sticta isidioimpresula; la considerable cantidad de especies particulares de cada forófito y la prevalencia de especies tolerantes al disturbio tales como: Cladonia ceratophylla, Herpothallon rubrocinctum, Hypotrachyna costaricensis, $H$. osorioi, Parmotrema arnoldii y P. mellissii; como los principales impactos observados que son efecto del establecimiento de plantaciones de Pinus patula en los bosques de roble de la reserva Rogitama.

El porte del bosque nativo de Quercus humboldtii en la reserva Rogitama es el resultado del experimento de restauración que se ha llevado en combinación con la siembra de Pinus patula, Este mosaico de vegetación sirve como resguardo de especies umbrófilas y heliófilas, pero presenta espacios que mantienen algún grado de intervención. Sin embargo, la presencia de taxones de los géneros Coccocarpia, Erioderma y Sticta muestran un avance en la sucesión vegetal que permite la observación de estrategias de vida características de los bosques nativos. Lo anterior sugiere que buenas prácticas de manejo de las plantaciones o el mantenimiento de remanentes sin tala y entresaca de las mismas conservan algunas condiciones de los bosques nativos, que son favorables para los líquenes.
Se destaca la función que cumple la reserva Rogitama como resguardo de diversidad en el corredor ecológico Guantiva-La Rusia-Iguaque y el rol como especies sombrilla que asumen los robles allí presentes, que no solo son importantes para el establecimiento de aves como Macroagelaius subalaris y Coeligena prunellei, sino que, particularmente para el caso de los líquenes, permiten el desarrollo de 161 especies epífitas (10\% de las especies registradas para Colombia).

\section{AGRADECIMIENTOS}

Los autores agradecen a Roberto Chavarro Chavarro y su familia, propietarios de la Reserva ROGITAMA BIODIVERSIDAD, por permitir el acceso a sus instalaciones y facilitar el apoyo logístico. Al Herbario UPTC por facilitar los equipos para el ascenso a dosel. A Fabio Simijaca y Fabián Simijaca por su acompañamiento en campo. A los biólogos Oscar Ruiz Rincón y Alejandra Palacios Rueda, por su apoyo en la recolección y curaduría del material vegetal. Al personal del herbario UDBC facilitar sus instalaciones para la determinación del material vegetal y a los investigadores del Grupo Colombiano de Liquenología (GCOL) por su asesoría en la determinación de diferentes grupos. Al Dr. Harrie Sipman del BGBM por su asesoría en la determinación del género Hypotrachyna, al biólogo David Díaz Escandón por su asesoría en la determinación del género Heterodermia. A la Dra. Angela Parrado por sus aportes en la elaboración del documento.

\section{CONFLICTO DE INTERESES}

Los autores declaran no tener conflicto de intereses.

\section{CONTRIBUCIÓN POR AUTOR}

Los autores son los únicos responsables de la obra en todos los aspectos que condujeron a la elaboración de su publicación. 


\section{REFERENCIAS BIBLIOGRÁFICAS}

Aguirre-C, J. (2008). Diversidad y riqueza de los líquenes en la región natural andina o sistema cordillerano. En J. O. Rangel-Ch. (ed.), Colombia diversidad biótica VI Riqueza y diversidad de los musgos y líquenes en Colombia (pp. 337-382). Bogotá D.C.: Instituto de Ciencias Naturales-Universidad Nacional de Colombia.

Alzate, F., Cardona, F. y Callejas, R. (2001). Diversidad y composición de epífitas vasculares en robledales de Antioquia (Colombia). Actualidades Biológicas, 23(74), 25-31.

Ardila, A. I., Moncada, B. y Lücking, R. (2014). Epiphyte homogenization and de-diversification on alien Eucalyptus versus native Quercus forest in the Colombian Andes: a case study using lirellate Graphidaceae lichens. Biodiversity and Conservation, 24(5), 1239-1252. DOI: https://doi.org/10.1007/ s10531-014-0855-7

Ariza Cortés, W., Huertas García, C., Hernández Ortiz, A., Geltvez Bernal, J., González Rodríguez, J. y López Gutiérrez, L. (2010). Caracterización y usos tradicionales de productos forestales no madeirables (PFNM) en el corredor de conservación Guantiva-La Rusia-Iguaque. Colombia Forestal, 13(1), 117-140.

Avella, A. y Cárdenas, L. M. (2010). Conservación y uso sostenible de los bosques de roble en el corredor de conservación Guantiva-La Rusia-Iguaque, departamentos de Santander y Boyacá, Colombia. Colombia Forestal, 13(1), 5-26. DOI: https://doi.org/10.14483/udistrital.jour.colomb. for.2010.1.a01

Ávila, F., Ángel, S. y López, R. (2010). Diversity and structure of an oak community in the Cachalú Biological Reserve, Encino (Santander-Colombia). Colombia Forestal, 13(1), 87-116. DOI: https://doi. org/10.14483/udistrital.jour.colomb.for.2010.1.a04

Bäcklund, S., Jönsson, M., Strengbom, J., Frisch, A. y Thor, G. (2016). A Pine Is a Pine and a Spruce Is a Spruce. The Effect of Tree Species and Stand Age on Epiphytic Lichen Communities. Plos One, 11(1), 1-18. DOI: https://doi.org/10.1371/journal. pone.0147004
Barlow, J., Gardner, T. A, Araujo, I. S., Ávila-Pires, T. C., Bonaldo, A. B., Costa, J. E., ... Peres, C. A. (2007). Quantifying the biodiversity value of tropical primary, secondary, and plantation forests. Proceedings of the National Academy of Sciences of the United States of America, 104(47), 18555-18560. DOI: https://doi.org/10.1073/pnas.0703333104

Berg, K., Hunt, S. L. y Gordon, A. M. (2013). Epiphytic lichens in jack pine and black spruce plantations in northern Ontario, Canada: diversity and community patterns. The Bryologist, 116(1), 65-77. DOI: https://doi.org/10.1639/0007-2745-116.1.065

Braun, A., Troeger, D., García, R., Aguayo, M., Barra, R. y Vogt, J. (2017). Assessing the impact of plantation forestry on plant biodiversity A comparison of sites in Central Chile and Chilean Patagonia. Global Ecology and Conservation, 10, 159-172. DOI: https://doi.org/10.1016/j.gecco.2017.03.006

Caceres, M. E. S., Lücking, R. y Rambold, G. (2007). Phorophyte specificity and environmental parameters versus stochasticity as determinants for species composition of corticolous crustose lichen communities in the Atlantic rain forest of northeastern Brazil. Mycological Progress, 6(3), 117-136. DOI: https://doi.org/10.1007/s11557-007-0532-2

Calviño-Cancela, M. (2013). Effectiveness of eucalypt plantations as a surrogate habitat for birds. Forest Ecology and Management, 310, 692-699. DOI: https://doi.org/10.1016/j.foreco.2013.09.014

Calviño-Cancela, M., López de Silanes, M. E., Rubido-Bará, M. y Uribarri, J. (2013). The potential role of tree plantations in providing habitat for lichen epiphytes. Forest Ecology and Management, 291, 386-395. DOI: https://doi.org/10.1016/j. foreco.2012.11.023

Calviño-Cancela, M., Rubido-Bará, M. y van Etten, E. J. B. (2012). Do eucalypt plantations provide habitat for native forest biodiversity? Forest Ecology and Management, 270, 153-162. DOI: https://doi. org/10.1016/j.foreco.2012.01.019

Chavarro, R. (2005). Ilustraciones y fotografías de aves-Coeligena prunellei Inca Negro- Black Inca (Príncipe de Arcabuco). Boletín SAO, 15(2), 118-122. 
Coca, L. F. y Sanín, D. (2010). Coccocarpia Pers. (Peltigerales-Ascomicetes Liquenizados) en Colombia. Tropical Bryology, 32, 19-38.

Cornelissen, J. y Ter Steege, H. (1989). Distribution and Ecology of Epiphytic Bryophytes and Lichens in Dry Evergreen Forest of Guyana. Journal of Tropical ECOlogy, 5(2), 131-150. DOI: https://doi.org/10.1017/ S0266467400003400

Corporación Autónoma Regional de Santander (CAS) (2006). Estudio técnico para efectos de establecer áreas susceptibles de aprovechamientos forestales persistentes de impacto reducido para la especie roble (Quercus humboldtii) de acuerdo con la resolución 0096 de 2006. Documento N. CAS-ROBLES-JAGR-00395-06-02.

Dingová Košuthová, A. y Šibík, J. (2013). Ecological indicator values and life history traits of terricolous lichens of the Western Carpathians. Ecological Indicators, 34, 246-259. DOI: https://doi.org/10.1016/j. ecolind.2013.05.013

Ellis, C. J. (2012). Lichen epiphyte diversity: A species, community and trait-based review. Perspectives in Plant Ecology, Evolution and Systematics, 14(2), 131-152. DOI: https://doi.org/10.1016/j. ppees.2011.10.001

Estrabou, C. (2007). Preferencia de forofito por los líquenes en el bosque chaqueño oriental. Bosque, 28(1), 46-49. DOI: https://doi.org/10.4067/ S0717-92002007000100007

Frazer, G. W., Canham, C. D. y Lertzman, K. P. (2000). Gap Light Analyzer (GLA), Version 2.0. Technological tools. Geological Society of America Bulletin, 191-197

Gasca, H. e Higuera, D. (2008). Artrópodos asociados al dosel de un robledal de Quercus humboldtii Bonpl. (Fagaceae) de la reserva Bosque Macanal (Bojacá, Colombia). Boletín de la Sociedad Entomológica Aragonesa, 43, 173-185.

Gil, J. y Morales-Puentes, M. (2014). Estratificación vertical de briófitos epífitos encontrados en Quercus humboldtii (Fagaceae) de Boyacá, Colombia. Revista de Biología Tropical, 62, 719-727.

Gil-Leguizamón, P., Morales-Puentes, M. y Díaz-Pérez, C. (2014). Population Assessment and Degree of Threat of Chalybea macrocarpa (Melastomataceae) Endemic Species from Colombia. Acta Biológica Colombiana, 19(2), 261-270. DOI: https://doi. org/10.15446/abc.v19n2.40691

Giordani, P., Brunialti, G., Bacaro, G. y Nascimbene, J. (2012). Functional traits of epiphytic lichens as potential indicators of environmental conditions in forest ecosystems. Ecological Indicators, 18, 413-420. DOI: https://doi.org/10.1016/j. ecolind.2011.12.006

Goward, T. y Arsenault, A. (2000). Cyanolichens and conifers: implications for global conservation. Forest Snow and Landscape Research, 75, 303-318.

Gradstein, S. R., Nadkarni, N. M., Krömer, T., Holz, I. y Nöske, N. (2003). A protocol for rapid and representative sampling of vascular and non-vascular epiphyte diversity of tropical rain forest. Selbyana, 24(1), 105-111.

Hauck, M. (2011). Site factors controlling epiphytic lichen abundance in northern coniferous forests. Flora-Morphology, Distribution, Functional ECology of Plants, 206(2), 81-90. DOI: https://doi.org/10.1016/j.flora.2010.02.001

Hauck, M. y Javkhlan, S. (2009). Epiphytic lichen diversity and its dependence on bark chemistry in the northern Mongolian dark taiga. Flora-Morphology, Distribution, Functional Ecology of Plants, 204(4), 278-288. DOI: https://doi.org/10.1016/j. flora.2008.03.001

Hietz-Seifert, U., Hietz, P. y Guevara, S. (1996). Epiphyte vegetation and diversity on remnant trees after forest clearance in southern Veracruz, Mexico. Biological Conservation, 75(2), 103-111. DOI: https://doi.org/10.1016/0006-3207(95)00071-2

Johansson, D. (1974). Ecology of vascular epiphytes in West African rain forest. Acta Phytogeogr. Suecica, 59, 1-141.

Käffer, M. I., Ganade, G. y Marcelli, M. P. (2009). Lichen diversity and composition in Araucaria forests and tree monocultures in southern Brazil. Biodiversity and Conservation, 18(13), 3543-3561. DOI: https://doi.org/10.1007/s10531-009-9659-6

Kapelle, M. (2006). Ecology and conservation of neotropical montanre Oak Forest (vol. 185). 
Berlin: Springer Verlag. DOI: https://doi. org/10.1007/3-540-28909-7

Koch Mossmann, N., Martins, S. M. de A., Lucheta, F. y Müller, S. C. (2013). Functional diversity and traits assembly patterns of lichens as indicators of successional stages in a tropical rainforest. Ecological Indicators, 34, 22-30. DOI: https://doi.org/10.1016/j. ecolind.2013.04.012

Komposch, H. y Hafellner, J. (2000). Diversity and vertical distribution of lichens in a venezuelan tropical lowland rain forest. Selbyana, 21(1,2), 11-24.

Kricke, R. (2002). Measuring bark pH. En: P. L. Nimis, C. Scheidegger y P. A. Wolseley (eds.), Monitoring with Lichens-Monitoring Lichens. Nato science series. IV. Earth and environmental sciences (pp. 333-336). Dordrecht: Kluwer Academic Publishers. DOI: https://doi.org/10.1007/978-94-010-0423-7_30

Lakatos, M., Rascher, U. y Büdel, B. (2006). Functional characteristics of corticolous lichens in the understory of a tropical lowland rain forest. New Phytologist, 172(4), 679-695. DOI: https://doi. org/10.1111/j.1469-8137.2006.01871.x

Llop, E., Pinho, P., Matos, P., Pereira, M. J. y Branquinho, C. (2012). The use of lichen functional groups as indicators of air quality in a Mediterranean urban environment. Ecological Indicators, 13(1), 215-221. DOI: https://doi.org/10.1016/j. ecolind.2011.06.005

Lowman, M., Moffett, M. y Rinker, B. (1993). A new technique for taxonomic and ecological sampling in rain forest canopies. Sebyana, 14, 75-79.

Lücking, R. (1999). Ecology of foliicolous lichens at the "Botarrama" trail (Costa Rica), a neotropical rain forest. I. Species composition and its ecogeographical implications. Biotropica, 31(4), 553-564. DOI: https://doi.org/10.1111/j.1744-7429.1999.tb00402.x

Lücking, R., Aptroot, A., Chaves, J. L., Sipman, H. J. M. y Umaña, L. (2008). A First Assessment of the Ticolichen Biodiversity Inventory in Costa Rica: The Genus Graphis, with Notes on the Genus Hemithecium (Ascomycota: Ostropales: Graphidaceae). Fieldiana Botany, 46, 1-126. DOI: https://doi.org/10.3158/0015-0746(2008)46[1:AFAOTT]2.0. $\mathrm{CO} ; 2$
Lücking, R., Dal Forno, M., Moncada, B., Coca, L. F., Vargas-Mendoza, L. Y., Aptroot, A., y Lawrey, J. (2016). Turbo-taxonomy to assemble a megadiverse lichen genus: seventy new species of Cora (Basidiomycota: Agaricales: Hygrophoraceae), honouring David Leslie Hawksworth's seventieth birthday. Fungal Diversity, 1-69. DOI: https://doi. org/10.1007/s13225-016-0374-9

Maestre, F. T., Bowker, M. A., Cantón, Y., Castillo-Monroy, A. P., Cortina, J., Escolar, C., Martínez, I. (2011). Ecology and functional roles of biological soil crusts in semi-arid ecosystems of Spain. Journal of Arid Environments, 75(12), 1282-1291. DOI: https://doi.org/10.1016/j.jaridenv.2010.12.008

Magurran, A. (1988). Ecological Diversity and Its Measurement. Nueva Jersey: Princeton University Press. DOI: https://doi.org/10.1007/978-94-015-7358-0

Marín-Corba, C. y Betancur, J. (1997). Estudio florístico en un robledal del santuario de flora y fauna de Iguaque (Boyacá-Colombia). Revista de la Academia Colombiana de Ciencias Exactas, Físicas y Naturales, 21(80), 249-259.

Martínez-Gil, A., Cadahía, E., Fernández de Simón, B., Gutiérrez-Gamboa, G., Nevares, I. y Del Alamo-Sanza, M. (2017). Quercus humboldtii (Colombian oak): characterization of oak heartwood phenolic composition with respect to traditional oak woods in oenology. Ciência e Técnica Vitivinícola, 32(2), 93-101. DOI: https://doi.org/10.1051/ ctv/20173202093

McCune, B., Grace, J. y Urban, D. (2002). Analysis of ecological communities. Portland, Oregon: MjM Software design.

Medina, M. (2010). Uso de especies forestales asociadas a bosques de roble en tres veredas del municipio de Encino-Santander. Colombia Forestal, 13(2), 237-244. DOI: https://doi.org/10.14483/udistrital. jour.colomb.for.2010.2.a04

Mercado-Díaz, J. A., Gould, W. A., González, G. y Lücking, R. (2015). Lichens in Puerto Rico: An Ecosystem Approach. San Juan, Puerto Rico. DOI: https:// doi.org/10.2737/IITF-GTR-46

Moncada, B. (2012). El género Sticta (Schreb.) Ach. en Colombia: taxonomía, ecogeografía e importancia. 
Bogotá: Universidad Nacional de Colombia. DOI: https://doi.org/10.1639/0007-2745-116.2.169

Moncada, B. y Forero, E. (2006). El género Pseudocyphellaria Vain. (Lobariaceae-Lichenized) Ascomycetes en Colombia. Caldasia, 28(2), 197-215.

Moncada, B., Coca, L. F. y Lücking, R. (2013). Neotropical members of Sticta (lichenized Ascomycota: Lobariaceae) forming photosymbiodemes, with the description of seven new species. The Bryologist, 116(2), 169-200. DOI: https://doi. org/10.1639/0007-2745-116.2.169

Morales, M., Otero, J., Van der Hammen, T., Cadena, C., Pedraza, C., Rodríguez, N. y Cárdenas, L. (2007). Atlas de páramos de Colombia. Bogotá D.C.: Instituto de Investigación de Recursos Biológicos Alexander von Humboldt.

Mossmann, N., Weidner, R. y Martins, S. (2012). Comunidade de liquens foliosos em Piptocarpha angustifolia Dusén ex Malme (Asteraceae) em área de Floresta Ombrófila Mista no estado do Rio Grande do Sul, Brasil. Iheringia Serie Botanica, 67(1), 47-57.

Nascimbene, J., Thor, G. y Nimis, P. L. (2013). Effects of forest management on epiphytic lichens in temperate deciduous forests of Europe-A review. Forest Ecology and Management, 298, 27-38. DOI: https://doi.org/10.1016/j.foreco.2013.03.008

Organización de las Naciones Unidas para la Alimentación y la Agricultura (FAO) (2002). Estado de la información forestal en Colombia. Santiago de Chile: FAO. Recuperado de ftp://ftp.fao.org/docrep/ fao/006/AD392S/AD392S00.pdf

Otálora, A. (2003). Mamíferos de los bosques de roble. Acta Biológica Colombiana, 8(2), 57-71.

Parada, J. A. (2015). On the description of the male of Pseudopogonogaster iguaquensis Salazar \& Carrejo, 2002 (Insecta:Mantodea:Thespidae). Boletín Científico. Centro de Museos. Museo de Historia Natural, 19(1), 258-262. DOI: https://doi. org/10.17151/bccm.2015.19.1.19

Pulido, K. y Ramos, C. (2016). Efecto de borde en la distribución de líquenes y el contenido de clorofilas en fragmentos de Polylepis quadrijuga (Rosaceae) en el páramo de La Rusia (Boyacá-Colombia). Revista de Biología Tropical, 64(4), 1683-1697. DOI: https://doi.org/10.15517/rbt.v64i4.22735

Ramírez, H. y Pérez, W. (2007). Mamíferos de un fragmento de bosque de roble en el departamento del Cauca, Colombia. Boletín Científico Centro de Museos Museo de Historia Natural Universidad de Caldas, 11, 65-79.

Ramírez-Morán, N. A., León-Gómez, M. y Lücking, R. (2016). Uso de biotipos de líquenes como bioindicadores de perturbación en fragmentos de bosque altoandino (reserva biológica "Encenillo", Colombia). Caldasia, 38(1), 31-52. DOI: https://doi. org/10.15446/caldasia.v38n1.57821

Rivas Plata, E., Lücking, R. y Lumbsch, H. T. (2007). When family matters: an analysis of Thelotremataceae (Lichenized Ascomycota: Ostropales) as bioindicators of ecological continuity in tropical forests. Biodiversity and Conservation, 17(6), 1319-1351. DOI: https://doi.org/10.1007/s10531-007-9289-9

Rosabal, D., Burgaz, A. R. y Reyes, O. J. (2013). Substrate preferences and phorophyte specificity of corticolous lichens on five tree species of the montane rainforest of Gran Piedra, Santiago de Cuba. The Bryologist, 116(2), 113-121. DOI: https://doi. org/10.1639/0007-2745-116.2.113

Rosabal, D., Burgaz, A.R., Altamirano, A. y Aragón, G. (2012). Differences in diversity of corticolous lichens between interior and edge of the Monte Barranca semi-deciduous forest, Santiago de Cuba. The Briologist, 115(2), 333-340. DOI: https://doi. org/10.1639/0007-2745-115.2.333

Rubiano, J. y Chaparro, M. (2006). Delimitación de áreas de isocontaminación atmosférica en el campus de la Universidad Nacional de Colombia mediante el análisis de bioindicadores (líquenes epífitos). Acta Biológica Colombiana, 11(2), 82-102.

Sáenz-Jiménez, F. (2010). An aproximation to the fauna associated with oak forests of Guantiva-La Rusia-Iguaque corridor (Boyacá-Santander, Colombia). Colombia Forestal, 13(2), 299-334. DOI: https://doi.org/10.14483/udistrital.jour.colomb. for.2010.2.a08 
Simijaca, D. (2011). Líquenes epífitos de Quercus humboldtii en el parque natural municipal Robledales de Tipacoque (Boyacá-Colombia) (trabajo de pregrado, Ciencias Biológicas). Universidad Pedagógica y Tecnológica de Colombia, Bogotá.

Simijaca, D. F., Vargas, D. L. y Morales, M. E. (2014). Uso de organismos vegetales no vasculares como indicadores de contaminación atmosférica urbana (Tunja, Boyacá, Colombia). Acta Biológica Colombiana, 19(2). DOI: https://doi.org/10.15446/abc. v19n2.40681

Sipman, H. J. M. y Aguirre-C., J. (1982). Contribución al conocimiento de los líquenes de Colombia -I. Clave genérica para los líquenes foliosos y fruticosos de los páramos colombianos. Caldasia, 13(64), 603-634.

Song, L., Liu, W. Y. y Nadkarni, N. M. (2012). Response of non-vascular epiphytes to simulated climate change in a montane moist evergreen broad-leaved forest in southwest China. Biological Conservation, 152, 127-135. DOI: https://doi.org/10.1016/j. biocon.2012.04.002

Soto, E., Lücking, R. y Rojas, A. B. (2012). Especificidad de forófito y preferencias microambientales de los líquenes cortícolas en cinco forófitos del bosque premontano de finca Zíngara, Cali, Colombia. Revista de Biología Tropical, 60(2), 843-856.
Stofer, S., Bergamini, A., Aragón, G., Carvalho, P., Coppins, B. J., Davey, S., ... Scheidegger, C. (2006). Species richness of lichen functional groups in relation to land use intensity. The Lichenologist, 38(4), 331. DOI: https://doi.org/10.1017/ S0024282906006207

Vanegas, S., Fagua, G. y Flórez, E. (2012). Distribución vertical de arañas asociadas a Quercus humboldtii y Clusia spp. en el santuario de Fauna y Flora Iguaque, Colombia. Acta Biológica Colombiana, 17(3), 635-656.

Vargas-Rojas, D. L. y Morales-Puentes, M. E. (2014). Hepáticas del Parque Natural Municipal "Robledales de Tipacoque", Boyacá-Colombia. Universitas Scientiarum, 19(3), 201-211. DOI: https://doi. org/10.11144/Javeriana.SC19-3.hpnm

Wolf, J. (1993a). Diversity paterns and biomass of epiphytic bryophytes and lichens along an altitudinal gradient in the northern Andes. Annals of the Missouri Botanical Garden, 80(4), 928-960. DOI: https://doi.org/10.2307/2399938

Wolf, J. (1993b). Epiphyte communities of tropical montane rain forest in the northern Andes II. Uper montane communities. Phytocoenologia, 22(1), 53-103. DOI: https://doi.org/10.1127/phyto/22/1993/53 y https://doi.org/10.1127/phyto/22/1993/1 
Anexo 1. Taxones registrados sobre Quercus humboldtii y Pinus patula en la reserva Rogitama Biodiversidad. Se presenta la frecuencia, los nuevos registros para Colombia (COL) y Boyacá (BOY) y las nuevas especies (sp, nov.).

\begin{tabular}{|c|c|c|c|c|c|c|}
\hline Familia & Especies & Pino & Roble & $\mathrm{COL}$ & BOY & sp. nov. \\
\hline Arthoniaceae & Herpothallon antillarum & 1 & 2 & 1 & 1 & 0 \\
\hline Arthoniaceae & Herpothallon aff. rubrocinctum & 1 & 13 & 1 & 1 & 1 \\
\hline Arthoniaceae & Herpothallon echinatum & 2 & 0 & 1 & 1 & 0 \\
\hline Arthoniaceae & Herpothallon granulare & 0 & 1 & 1 & 1 & 0 \\
\hline Arthoniaceae & Herpothallon japonicum & 0 & 1 & 1 & 1 & 0 \\
\hline Arthoniaceae & Herpothallon mínimum & 1 & 2 & 0 & 1 & 0 \\
\hline Arthoniaceae & Herpothallon roseocinctum & 1 & 0 & 0 & 1 & 0 \\
\hline Arthoniaceae & Herpothallon rubrocinctum & 28 & 26 & 0 & 0 & 0 \\
\hline Catillariaceae & Catillaria sp.1 & 0 & 2 & 0 & 1 & 0 \\
\hline Cladoniaceae & Cladonia ceratophylla & 14 & 20 & 0 & 0 & 0 \\
\hline Cladoniaceae & Cladonia chlorophaea & 1 & 4 & 0 & 0 & 0 \\
\hline Cladoniaceae & Cladonia didyma & 5 & 8 & 0 & 0 & 0 \\
\hline Cladoniaceae & Cladonia pyxidata & 4 & 0 & 0 & 1 & 0 \\
\hline Coccocarpiaceae & Coccocarpia palmicola & 9 & 7 & 0 & 0 & 0 \\
\hline Coccocarpiaceae & Coccocarpia prostrata & 5 & 0 & 0 & 1 & 0 \\
\hline Coenogoniaceae & Coenogonium isidiiferum & 3 & 1 & 1 & 1 & 0 \\
\hline Coenogoniaceae & Coenogonium luteum & 1 & 1 & 0 & 0 & 0 \\
\hline Coenogoniaceae & Coenogonium nepalense & 0 & 1 & 1 & 1 & 0 \\
\hline Coenogoniaceae & Coenogonium roumeguerianum & 4 & 0 & 1 & 1 & 0 \\
\hline Coenogoniaceae & Coenogonium sp.1 & 0 & 4 & 0 & 0 & 0 \\
\hline Coenogoniaceae & Coenogonium stenosporum & 0 & 2 & 0 & 1 & 0 \\
\hline Collemataceae & Leptogium diaphanum & 0 & 2 & 0 & 1 & 0 \\
\hline Crysotricaceae & Chrysotrix candelaris & 5 & 0 & 0 & 0 & 0 \\
\hline Graphidaceae & Fissurina sp. 1 & 0 & 5 & 0 & 0 & 0 \\
\hline Graphidaceae & Fissurina triticea & 3 & 0 & 0 & 1 & 0 \\
\hline Graphidaceae & Graphis adpresa & 1 & 1 & 0 & 1 & 0 \\
\hline Graphidaceae & Graphis aff. subtracta & 1 & 0 & 0 & 0 & 0 \\
\hline Graphidaceae & Graphis bifera & 0 & 1 & 1 & 1 & 0 \\
\hline Graphidaceae & Graphis cognata & 2 & 0 & 1 & 1 & 0 \\
\hline Graphidaceae & Graphis conferta & 5 & 12 & 1 & 1 & 0 \\
\hline Graphidaceae & Graphis conspersa & 0 & 2 & 1 & 1 & 0 \\
\hline Graphidaceae & Graphis dracaenae & 2 & 0 & 1 & 1 & 0 \\
\hline Graphidaceae & Graphis duplicata & 0 & 1 & 0 & 1 & 0 \\
\hline Graphidaceae & Graphis elegans & 6 & 0 & 0 & 0 & 0 \\
\hline Graphidaceae & Graphis emersa & 1 & 0 & 1 & 1 & 0 \\
\hline Graphidaceae & Graphis nana & 1 & 3 & 1 & 1 & 0 \\
\hline Graphidaceae & Graphis nanodes & 0 & 1 & 0 & 1 & 0 \\
\hline Graphidaceae & Graphis nuda & 1 & 3 & 0 & 1 & 0 \\
\hline Graphidaceae & Graphis ovata & 5 & 1 & 1 & 1 & 0 \\
\hline
\end{tabular}




\begin{tabular}{|c|c|c|c|c|c|c|}
\hline Familia & Especies & Pino & Roble & COL & BOY & sp. nov. \\
\hline Graphidaceae & Graphis pedunculata & 1 & 0 & 1 & 1 & 0 \\
\hline Graphidaceae & Graphis phaeospora & 1 & 3 & 1 & 1 & 0 \\
\hline Graphidaceae & Graphis rhizocola & 0 & 1 & 0 & 0 & 0 \\
\hline Graphidaceae & Graphis ruiziana & 0 & 1 & 0 & 1 & 0 \\
\hline Graphidaceae & Graphis sp.1 & 0 & 2 & 0 & 0 & 0 \\
\hline Graphidaceae & Graphis subtracta & 2 & 0 & 0 & 1 & 0 \\
\hline Graphidaceae & Graphis tenella & 1 & 0 & 0 & 1 & 0 \\
\hline Graphidaceae & Hemithecium sp1 & 0 & 1 & 0 & 0 & 0 \\
\hline Graphidaceae & Ocellularia buckii & 2 & 1 & 1 & 1 & 0 \\
\hline Graphidaceae & Ocellularia sp. & 1 & 11 & 0 & 0 & 0 \\
\hline Graphidaceae & Phaeographis inconspicua & 4 & 0 & 1 & 1 & 0 \\
\hline Graphidaceae & Phaeographis major & 0 & 3 & 1 & 1 & 0 \\
\hline Graphidaceae & Platythecium leiogramma & 0 & 1 & 0 & 1 & 0 \\
\hline Hygrophoraceae & Cora glabrata & 1 & 0 & 0 & 0 & 0 \\
\hline Hygrophoraceae & Cora sp.1 & 1 & 1 & 0 & 0 & 0 \\
\hline Hygrophoraceae & Cora sp.2 & 1 & 0 & 0 & 0 & 0 \\
\hline Hygrophoraceae & Dictyonema obscuratum & 2 & 4 & 0 & 1 & 0 \\
\hline Lecanoraceae & Lecanora sp & 4 & 2 & 0 & 0 & 0 \\
\hline Lobariaceae & Crocodia aurata & 1 & 0 & 0 & 0 & 0 \\
\hline Lobariaceae & Sticta colombiana & 1 & 21 & 0 & 0 & 0 \\
\hline Lobariaceae & Sticta isidioimpressula & 0 & 4 & 0 & 0 & 0 \\
\hline Megalariaceae & Lopezaria versicolor & 1 & 2 & 0 & 1 & 0 \\
\hline Megalosporaceae & Bisoloma sp & 0 & 1 & 0 & 0 & 0 \\
\hline Megalosporaceae & Megalospora kalbii & 0 & 1 & 1 & 1 & 0 \\
\hline Megalosporaceae & Megalospora tuberculosa & 1 & 0 & 0 & 1 & 0 \\
\hline Pannariaceae & Erioderma sorediatum & 1 & 0 & 0 & 1 & 0 \\
\hline Parmeliaceae & Anzia leucobatoides & 0 & 2 & 1 & 1 & 0 \\
\hline Parmeliaceae & Canoparmelia caroliniana & 7 & 1 & 0 & 0 & 0 \\
\hline Parmeliaceae & Hypotrachyna vexans & 1 & 4 & 0 & 0 & 0 \\
\hline Parmeliaceae & Hypotrachyna aff. paramensis & 1 & 0 & 0 & 0 & 0 \\
\hline Parmeliaceae & Hypotrachyna bogotensis & 0 & 24 & 0 & 0 & 0 \\
\hline Parmeliaceae & Hypotrachyna chlorina & 0 & 11 & 0 & 1 & 0 \\
\hline Parmeliaceae & Hypotrachyna costaricensis & 8 & 8 & 0 & 1 & 0 \\
\hline Parmeliaceae & Hypotrachyna dactylifera & 2 & 4 & 0 & 1 & 0 \\
\hline Parmeliaceae & Hypotrachyna degelii & 1 & 0 & 0 & 1 & 0 \\
\hline Parmeliaceae & Hypotrachyna densirhizinata & 0 & 1 & 0 & 0 & 0 \\
\hline Parmeliaceae & Hypotrachyna dentella & 1 & 2 & 0 & 1 & 0 \\
\hline Parmeliaceae & Hypotrachyna elongata & 1 & 0 & 1 & 1 & 0 \\
\hline Parmeliaceae & Hypotrachyna endochlora & 1 & 0 & 1 & 1 & 0 \\
\hline Parmeliaceae & Hypotrachyna ensifolia & 2 & 5 & 0 & 0 & 0 \\
\hline Parmeliaceae & Hypotrachyna gondylophora & 1 & 0 & 0 & 0 & 0 \\
\hline Parmeliaceae & Hypotrachyna imbricatula & 6 & 2 & 0 & 1 & 0 \\
\hline Parmeliaceae & Hypotrachyna immaculata & 17 & 2 & 0 & 1 & 0 \\
\hline Parmeliaceae & Hypotrachyna laevigata & 11 & 2 & 0 & 0 & 0 \\
\hline
\end{tabular}




\begin{tabular}{|c|c|c|c|c|c|c|}
\hline Familia & Especies & Pino & Roble & COL & BOY & sp. nov. \\
\hline Parmeliaceae & Hypotrachyna lividescens & 2 & 0 & 0 & 0 & 0 \\
\hline Parmeliaceae & Hypotrachyna minuscula & 2 & 0 & 1 & 1 & 0 \\
\hline Parmeliaceae & Hypotrachyna neodissecta & 0 & 2 & 1 & 1 & 0 \\
\hline Parmeliaceae & Hypotrachyna osorioi & 8 & 9 & 1 & 1 & 0 \\
\hline Parmeliaceae & Hypotrachyna osseoalba & 8 & 0 & 0 & 1 & 0 \\
\hline Parmeliaceae & Hypotrachyna producta & 5 & 1 & 0 & 1 & 0 \\
\hline Parmeliaceae & Hypotrachyna prolongata & 3 & 1 & 0 & 1 & 0 \\
\hline Parmeliaceae & Hypotrachyna protoformosana & 2 & 0 & 1 & 1 & 0 \\
\hline Parmeliaceae & Hypotrachyna pseudosinuosa & 11 & 1 & 0 & 1 & 0 \\
\hline Parmeliaceae & Hypotrachyna pulvinata & 1 & 0 & 0 & 0 & 0 \\
\hline Parmeliaceae & Hypotrachyna revoluta & 5 & 1 & 0 & 1 & 0 \\
\hline Parmeliaceae & Hypotrachyna sanjosensis & 1 & 0 & 1 & 1 & 0 \\
\hline Parmeliaceae & Hypotrachyna stictifera & 3 & 0 & 1 & 1 & 0 \\
\hline Parmeliaceae & Hypotrachyna sublaevigata & 1 & 0 & 1 & 1 & 0 \\
\hline Parmeliaceae & Hypotrachyna subpustulifera & 16 & 4 & 1 & 1 & 0 \\
\hline Parmeliaceae & Parmelinopsis horrescens & 18 & 2 & 0 & 0 & 0 \\
\hline Parmeliaceae & Parmelinopsis sp.2 & 4 & 1 & 0 & 0 & 0 \\
\hline Parmeliaceae & Parmotrema aff. horridum & 3 & 0 & 0 & 0 & 0 \\
\hline Parmeliaceae & Parmotrema arnoldii & 11 & 4 & 0 & 0 & 0 \\
\hline Parmeliaceae & Parmotrema bonplandii & 2 & 0 & 0 & 1 & 0 \\
\hline Parmeliaceae & Parmotrema cetratum & 1 & 7 & 0 & 0 & 0 \\
\hline Parmeliaceae & Parmotrema hababianum & 3 & 1 & 0 & 1 & 0 \\
\hline Parmeliaceae & Parmotrema hypoleucinum & 0 & 4 & 1 & 1 & 0 \\
\hline Parmeliaceae & Parmotrema indicum & 1 & 0 & 1 & 1 & 0 \\
\hline Parmeliaceae & Parmotrema internexum & 1 & 0 & 1 & 1 & 0 \\
\hline Parmeliaceae & Parmotrema mellissii & 44 & 5 & 0 & 1 & 0 \\
\hline Parmeliaceae & Parmotrema mirandum & 1 & 0 & 1 & 1 & 0 \\
\hline Parmeliaceae & Parmotrema rampoddense & 9 & 2 & 0 & 1 & 0 \\
\hline Parmeliaceae & Parmotrema reticulatum & 9 & 7 & 0 & 0 & 0 \\
\hline Parmeliaceae & Parmotrema robustum & 8 & 2 & 0 & 0 & 0 \\
\hline Parmeliaceae & Parmotrema simulans & 10 & 10 & 0 & 0 & 0 \\
\hline Parmeliaceae & Parmotrema stuppeum & 2 & 0 & 1 & 1 & 0 \\
\hline Parmeliaceae & Parmotrema subarnoldii & 9 & 4 & 1 & 1 & 0 \\
\hline Parmeliaceae & Parmotrema subisidiosum & 4 & 7 & 0 & 0 & 0 \\
\hline Parmeliaceae & Punctelia subrudecta & 2 & 0 & 0 & 0 & 0 \\
\hline Parmeliaceae & Usnea columbiana & 2 & 5 & 0 & 1 & 0 \\
\hline Parmeliaceae & Usnea firma & 1 & 0 & 1 & 1 & 0 \\
\hline Parmeliaceae & Usnea malmei & 1 & 0 & 1 & 1 & 0 \\
\hline Parmeliaceae & Usnea perhispidella & 1 & 0 & 1 & 1 & 0 \\
\hline Parmeliaceae & Usnea sancteritae & 11 & 8 & 1 & 1 & 0 \\
\hline Parmeliaceae & Usnea sp.1 & 0 & 2 & 0 & 0 & 0 \\
\hline Parmeliaceae & Usnea sp.2 & 0 & 1 & 0 & 0 & 0 \\
\hline Parmeliaceae & Usnea subscabrosa & 1 & 1 & 1 & 1 & 0 \\
\hline Peltigeraceae & Peltigera sp. & 0 & 1 & 0 & 0 & 0 \\
\hline
\end{tabular}




\begin{tabular}{|c|c|c|c|c|c|c|}
\hline Familia & Especies & Pino & Roble & COL & BOY & sp. nov. \\
\hline Pertusariaceae & Pertusaria sp.1 & 2 & 2 & 0 & 0 & 0 \\
\hline Pertusariaceae & Pertusaria sp.2 & 1 & 0 & 0 & 0 & 0 \\
\hline Pertusariaceae & Pertusaria sp.3 & 0 & 2 & 0 & 0 & 0 \\
\hline Pertusariaceae & Pertusaria sp.4 & 5 & 1 & 0 & 0 & 0 \\
\hline Pertusariaceae & Pertusaria sp.5 & 9 & 2 & 0 & 0 & 0 \\
\hline Pertusariaceae & Pertusaria sp.6 & 0 & 1 & 0 & 0 & 0 \\
\hline Pertusariaceae & Pertusaria sp.7 & 1 & 0 & 0 & 0 & 0 \\
\hline Pertusariaceae & Pertusaria sp.8 & 0 & 1 & 0 & 0 & 0 \\
\hline Physciaceae & Dirinaria sp. & 1 & 0 & 0 & 0 & 0 \\
\hline Physciaceae & Heterodermia boryi & 3 & 10 & 1 & 1 & 0 \\
\hline Physciaceae & Heterodermia casarettiana & 5 & 2 & 0 & 1 & 0 \\
\hline Physciaceae & Heterodermia corallophora & 0 & 1 & 0 & 0 & 0 \\
\hline Physciaceae & Heterodermia japonica & 19 & 7 & 0 & 1 & 0 \\
\hline Physciaceae & Heterodermia kurokawae & 1 & 0 & 0 & 1 & 0 \\
\hline Physciaceae & Heterodermia leucomela & 1 & 0 & 0 & 0 & 0 \\
\hline Physciaceae & Heterodermia lutescens & 1 & 10 & 0 & 0 & 0 \\
\hline Physciaceae & Heterodermia magellanica & 0 & 2 & 0 & 1 & 0 \\
\hline Physciaceae & Heterodermia obscurata & 1 & 1 & 0 & 0 & 0 \\
\hline Physciaceae & Heterodermia squamulosa & 2 & 2 & 0 & 0 & 0 \\
\hline Physciaceae & Heterodermia vulgaris & 0 & 1 & 0 & 1 & 0 \\
\hline Porinaceae & Porina nucula & 0 & 1 & 0 & 1 & 0 \\
\hline Porinaceae & Porina $s p$ & 1 & 0 & 0 & 0 & 0 \\
\hline Pyrenulaceae & Pyrenula bahiana & 0 & 3 & 0 & 1 & 0 \\
\hline Pyrenulaceae & Pyrenula microcarpa & 1 & 0 & 0 & 1 & 0 \\
\hline Pyrenulaceae & Pyrenula sp.1 & 0 & 1 & 0 & 0 & 0 \\
\hline Ramalinaceae & Bacidia auerswaldii & 2 & 0 & 1 & 1 & 0 \\
\hline Rocellaceae & Opegrapha sp. & 1 & 0 & 0 & 0 & 0 \\
\hline Rocellaceae & Opegrapha sp.1 & 1 & 8 & 0 & 0 & 0 \\
\hline Sphaerophoraceae & Bunodophoron sp1 & 0 & 3 & 0 & 0 & 0 \\
\hline Trypetheliaceae & Astrothelium aff. amylosporum & 0 & 2 & 1 & 1 & 1 \\
\hline Trypetheliaceae & Astrothelium aff. flavoduplex & 0 & 6 & 1 & 1 & 1 \\
\hline Trypetheliaceae & Astrothelium sp. & 0 & 1 & 0 & 0 & 0 \\
\hline Trypetheliaceae & Astrothelium sp.2 & 0 & 1 & 0 & 0 & 0 \\
\hline Trypetheliaceae & Bogoriella nonensis & 0 & 1 & 1 & 1 & 0 \\
\hline
\end{tabular}

\section{(c) $(1) \Theta(\Theta$}

Colombia Forestal • ISSN 0120-0739 • e-ISSN 2256-201X • Bogotá-Colombia • Vol. 21 No. 2 • pp. 123-141 